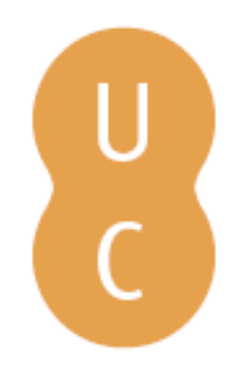

\title{
nombalina
}

\section{Baudelaire en situation: un lecteur de Chateaubriand aux carrefours de la modernité}

Autor(es): $\quad$ Laurel, Maria Hermínia Amado

Publicado por: Imprensa da Universidade de Coimbra

URL

persistente:

URI:http://hdl.handle.net/10316.2/38699

DOI:

DOI:ttp://dx.doi.org/10.14195/978-989-26-1164-8_22

Accessed : $\quad$ 26-Apr-2023 10:49:10

A navegação consulta e descarregamento dos títulos inseridos nas Bibliotecas Digitais UC Digitalis, UC Pombalina e UC Impactum, pressupõem a aceitação plena e sem reservas dos Termos e Condições de Uso destas Bibliotecas Digitais, disponíveis em https://digitalis.uc.pt/pt-pt/termos.

Conforme exposto nos referidos Termos e Condições de Uso, o descarregamento de títulos de acesso restrito requer uma licença válida de autorização devendo o utilizador aceder ao(s) documento(s) a partir de um endereço de IP da instituição detentora da supramencionada licença.

Ao utilizador é apenas permitido o descarregamento para uso pessoal, pelo que o emprego do(s) título(s) descarregado(s) para outro fim, designadamente comercial, carece de autorização do respetivo autor ou editor da obra.

Na medida em que todas as obras da UC Digitalis se encontram protegidas pelo Código do Direito de Autor e Direitos Conexos e demais legislação aplicável, toda a cópia, parcial ou total, deste documento, nos casos em que é legalmente admitida, deverá conter ou fazer-se acompanhar por este aviso. 
Maria Hermínia Amado Laurel

Universidade de Aveiro

\section{BAUDELAIRE EN SITUATION \\ UN LECTEUR DE CHATEAUBRIAND AUX CARREFOURS DE LA MODERNITÉ ${ }^{1}$}

l'œuvre est toujours en situation prophétique Roland Barthes, 1966.

La critique littéraire consacre traditionnellement Charles Baudelaire comme le premier des modernes. Elle s'intéresse moins, pourtant, aux filiations du poète et critique d'art dans le mouvement de la modernité naissante du XIX siècle. Or, parmi les écrivains qui ont marqué le mouvement des idées littéraires au début de ce siècle, Chateaubriand occupe une place de choix. Chateaubriand, prisé en son temps comme romancier, mémorialiste et penseur des révolutions, et Baudelaire, poète, traducteur d'Edgar Alan Poe, critique littéraire et auteur de tant d' écrits sur l'art, bien qu’à des moments historiques distincts, mais subséquents, soumettent la modernité à un questionnement qui s'avère une constante tout au long de leur production.

Dans un projet daté des années 80 du siècle dernier, Leyla Perrone-Moisés se proposait d'étudier de nouvelles formulations de l'histoire littéraire à partir des critères selon lesquels certains écrivains y avaient effectué leur sélection personnelle. Lectrice de Borges, elle soulignait que, dans le contexte d'une esthétique de la

\footnotetext{
1 Este trabalho é financiado por Fundos Nacionais através da FCT - Fundação para a Ciência e a Tecnologia no âmbito do projecto «PEst-OE/ELT/UI0500/2011».
} 
modernité, "é o presente que vai decidir o valor do passado" (1984: 10), dans la mesure où «O posterior permite a leitura de aspectos anteriormente invisíveis no anterior» (12).

Dans cette ligne de pensée, il nous a semblé pertinent de réfléchir à la manière dont l'œuvre de Baudelaire, lecteur de Chateaubriand, peut contribuer à resituer son prédécesseur dans l'histoire de la modernité littéraire, tout en se situant lui-même face à cette modernité.

Replacer l'œuvre de ces deux auteurs dans leur contexte historique sans pourtant l'y réduire me semble d'autant plus pertinent que nous avons affaire à Chateaubriand, considéré par Antoine Compagnon comme «le premier des antimodernes", dans son livre Les antimodernes (2005: 53) et à Baudelaire, qui, pour Claude Pichois, était le "dernier classique et tout à la fois le premier moderne» (Baudelaire, 1975: 791). Compagnon constate dans «'aventure intellectuelle et littéraire [française] des $\mathrm{XIX}^{\mathrm{e}}$ et $\mathrm{Xx}^{\mathrm{e}}$ siècles» la permanence de valeurs de stabilité, "à rebours du grand récit de la modernité battante et conquérante»(11).

Chateaubriand, qui a voulu comprendre l'histoire et ses contours d'ordre politique; Baudelaire, intéressé avant tout par la connaissance des «aspects de la nature et [d]es situations de l'homme, que les artistes du passé ont dédaignés ou n'ont pas connus», tel qu'il l'écrit dans son Salon de 1846 (1976: 421). Par ailleurs, la réflexion sur la place des deux auteurs cités dans l'histoire s'avère autrement nécessaire lorsqu'il s'agit de les situer par rapport à la modernité, concept, en lui-même, de nature historique. Comprise comme l'espace du présent, mais aussi comme l'espace de l'interpellation au futur, leur ouvre écrit sa propre situation historique $^{2}$ : en elle s'inscrivent des lectures plurielles de l'histoire qui la composent sans pour autant l'épuiser; elle confirme la nature prophétique des chefs d'ouvre ${ }^{3}$.

Chateaubriand et Baudelaire situent leur œuvre dans l'histoire; revenant, sur ce point, à la proposition de Compagnon dans le livre cité, nous dirions avec lui que, dans la mesure où leur œuvre résiste à l'histoire, ils sont modernes, parce qu'antimodernes. Tel que Compagnon le constate, "De Chateaubriand à Proust

2 Nous évoquons la réflexion de Sartre à propos de la "situation de l'écrivain en 1947", marquée par son inscription dans l'histoire contemporaine (Sartre, 1948: 203-374), plus particulièrement, les pages 247-374.

3 V., plus loin, n. 4, pour la référence implicite à la pensée de Barthes. 
au moins, entre le Génie du christianisme et À la recherche du temps perdu, en passant par Baudelaire et tant d'autres, le génie antimoderne s'est réfugié dans la littérature, et dans la littérature même que nous qualifions de moderne, dans la littérature dont la postérité a fait son canon, littérature dont la résistance idéologique est inséparable de son audace littéraire» (2005: 10).

Moderne, un concept qui change. Baudelaire en a conscience, qui qualifie l'œuvre de Chateaubriand de "réactionnaire», lorsqu'il cherche à se documenter pour un dialogue pour son projet dramaturgique Le Marquis du Ier housards, auquel il travailla entre 1859 et 1861, mais qui resta à l'état de canevas:

"(Ce dialogue, fort difficile à faire, [...] je le ferai avec des morceaux de la littérature réactionnaire du temps. Outre que j'en connais quelque chose [...] il faut voir les Mémoires de Chateaubriand, surtout.)»(1975: 637). C'est sans doute dans son Salon de 1846 que Baudelaire établit le plus clairement la filiation du romantisme à la modernité, héritier, en cela, du Stendhal de Racine et Shakespeare, pour qui était moderne l'œuvre qui avait le mérite de provoquer le plus de plaisir chez celui qui la lisait au moment où il la lisait:

«Le romanticisme est l'art de présenter aux peuples les œuvres littéraires qui, dans l'état actuel de leurs habitudes et de leurs croyances, sont susceptibles de leur donner le plus de plaisir possible» (Stendhal, 1925: 23). Pour le poète et critique d'art qu'il était, «s'appeler romantique et regarder systématiquement le passé, c'est se contredire» (Baudelaire, 1976: 420). D'autres facteurs se conjuguent encore dans sa définition du romantisme, à part l'appel de la contemporanéité: la cohérence entre le sentiment du poète et le choix des sujets artistiques, et le culte du beau. C'est dans ces termes que Baudelaire affirme le caractère éminemment subjectif de l'expression artistique: «Le romantisme n'est précisément ni dans le choix des sujets ni dans la vérité exacte, mais dans la manière de sentir» (420); il souligne le caractère esthétique du romantisme: "Pour moi, le romantisme est l'expression la plus récente, la plus actuelle du beau.» (420). Son esthétique allie modernité et sensibilité: "Qui dit romantisme dit art moderne, - c'est-à-dire intimité, spiritualité, couleur, aspiration vers l'infini». C'est pourquoi «le romantisme ne consistera pas dans une exécution parfaite, mais dans une conception analogue à la morale du siècle» (421). Une morale qui n'est sans doute pas très éloignée de la pensée de Madame de Staël sur le rapport dichotomique entre les littératures du Nord de l'Europe et celles du Sud, dont l'écho se fait entendre dans la filiation 
que Baudelaire attribue au romantisme: "Le romantisme est fils du Nord, et le Nord est coloriste [...] En revanche le Midi est naturaliste»(421).

Situées dans un entre-deux, les ouvres de Chateaubriand et de Baudelaire sont aussi antimodernes, non seulement parce qu'elles interrogent leur contemporanéité, et lui résistent, mais parce qu'elles annoncent d'autres voies à la modernité, qui seront comprises plus tard, à la distance de l'histoire, leurs œuvres étant en situation prophétique. Dans ce sens, elles contribuent à dater définitivement des ouvres considérées modernes à l'époque, ayant réussi à se constituer un large public: c'est le cas de Fanny, roman qu'Ernest Feydau publia au début de 1858 et fut «reçu par la critique comme un nouveau Madame Bovary" (Reverzy, 2009: 273).

Stendhal n'avait peut-être pas tort quand il affirmait écrire pour les 'happy few'...

Des œuvres qui se sont construites sur l'inachèvement ne sauraient susciter que des lectures conjecturales ${ }^{4}$, non définitives 5 .

Rappelons-nous le long parcours d'écriture et de réécriture de ses textes par Chateaubriand, comme c'est le cas de Mémoires d'outre-tombe, intitulé d'abord Mémoires de ma vie, ou bien la publication de ses œuvres complètes dès 1826, alors qu'il ne meurt qu'en 1848. De même chez Baudelaire: combien de lettres échangées entre lui et ses éditeurs avant qu'il ne leur envoie ses manuscrits à publier, qu'il veut encore retoucher, mais qu'il cache, refusant de leur montrer leur genèse; tant de projets restés à l'état d'ébauches ou de fragments; son goût du trait non achevé des dessins de Delacroix ou de Constantin Guys; le fait même de ne jamais avoir voulu rédiger de préfaces définitives, ce que confirme l'ambiguité de la lettre-dédicace à A. Houssaye que les éditeurs des Petits Poèmes en Prose situent en guise d'ouverture au recueil, en absence de texte introductif

\footnotetext{
4 Nous empruntons l'expression de Franco Moretti dans "Conjectures on world reading", New Left Review, Jan. Feb. 2000: 54-68.

5 La référence au rôle de la lecture, d'après Barthes, se révèle pertinente pour notre propos: «'œuvre n'est entourée, désignée, protégée, dirigée par aucune situation, aucune vie pratique n'est là pour nous dire le sens qu'il faut lui donner [...] elle possède quelque chose de la concision pythique, paroles conformes à un premier code (la Pythie ne divaguait pas) et cependant ouverte à plusieurs sens, car elles étaient prononcées hors de toute situation - sinon la situation même de l'ambiguité: l'œuvre est toujours en situation prophétique. Certes, en ajoutant ma situation à la lecture que je fais d'une œuvre, je puis réduire son ambiguïté [...]; mais cette situation, changeante, compose l'œuvre, elle ne la retrouve pas» (1994: 39).
} 
donné par Baudelaire à cette fin, ou encore son malaise devant la révélation que fut pour lui la traduction de The Philosophy of Composition, d' Edgar Alan Poe...

Attardons-nous sur les moments historiques vécus par Chateaubriand et par Charles Baudelaire.

La vie de Chateaubriand ${ }^{6}$ est marquée par la recherche d'un espace de bonheur entre le passé et le présent, un présent qu'il refuse, celui de la modernité, en quête d'une autre temporalité, qu'il localise dans des sociétés géographiquement distantes, en marge des modèles de civilisation qui sont les siens, régies par les traditions de leur passé tribal. C'est dans cet espace creux que s'affrontent les deux temporalités: le passé symbolique des 'sauvages', appartenant à une époque symboliquement primitive et le présent des colonisateurs, des déshérités qui affluent à ces contrées, ceux-là même que la modernité a rejetés hors du temps.

Des changements historiques profonds ont marqué la vie de Chateaubriand et celle de Baudelaire. Contemporain de l'avènement du nouveau monde - la naissance des Etats-Unis, dont l'histoire suit de près l'histoire de la France à l'époque -, Chateaubriand a été le témoin mais aussi l'acteur des grands bouleversements qu'a connus l'histoire de son pays depuis la fin de l'Ancien Régime jusqu'à l'avènement de la IIe République; pour Baudelaire l'expérience de ces changements s'est bien souvent traduite par des promesses non réalisées. Une civilisation nouvelle se répandait en Europe, dont la figure de proue était le bourgeois, et le dogme, le progrès, mythe du Second Empire. Ayant survécu de dix-neuf ans à son aîné, Baudelaire assiste au triomphe de la civilisation libérale, lui, le poète qui se voulait "dépolitiqué" après 1848, pour avoir reconnu son impuissance devant le cours de l'histoire. Son œuvre poétique, de même que ses articles, notamment ses Salons, révèlent le questionnement profond auquel il soumet les valeurs contemporaines, tout en se constituant comme l'espace privilégié de sa réflexion sur la création.

La contextualisation historique des deux auteurs est révélatrice d'une part, des lignes de rupture sociales, politiques et esthétiques entre deux mondes, d'autre part, de leur prise de conscience de l'enracinement historique de leur

${ }^{6}$ Pierre Barbéris, dans Chateaubriand: une réaction au monde moderne, propose une analyse marxiste des contours socio-politiques et économiques déterminants de la vie et de l'oeuvre de Chateaubriand. 
Moi. Constatant que "le vieil ordre européen expire», et situé face à un "avenir» qu'il lui est difficile de "comprendre», Chateaubriand revient, à la fin de ses Mémoires, sur la question des effets du progrès sur le "monde moral», dont la marche ne peut être comparable à celle du "monde matériel", domaine de la massification: "Mille cerveaux auront beau se coaliser, ils ne composeront jamais le chef-d'œuvre qui sort de la tête d'un Homère», conclut-il (1951, t. II: 923).

L'expérience de la désillusion rapproche Baudelaire et Chateaubriand. Quelques passages de leur oeuvre semblent se recouper, à tel point les deux écrivains ont souffert de ce «désert d'hommes» que constituait la société contemporaine. L'expression revient dans le discours de Baudelaire pour caractériser l'espace socio-urbain où évolue le peintre de la vie moderne, "toujours voyageant à travers le grand désert d'hommes» (1976: 694), qui partage avec René, «inconnu [mêlé] à la foule», sa singularité (1969: 127). La poursuite d'un idéal inatteignable les réunit, impuissants devant les «bornes» qui limitent leur quête de "profondes avenues à l'imagination». "Est-ce ma faute, si je trouve partout les bornes, si ce qui est fini n'a pour moi aucune valeur?» (128), s'interroge René, préludant à l'un des plus beaux poèmes en prose de Baudelaire, «L'Etranger»: «- J'aime les nuages... les nuages qui passent... là-bas... là-bas... les merveilleux nuages!» (1975: 277). Ce sentiment se présente à eux soit sous la forme de l'abîme insondable - et René le constate en faisant le bilan de sa vie: "C'est ainsi que toute ma vie j'ai eu devant les yeux une création à la fois immense et imperceptible, et un abîme ouvert à mes côtés" (1969: 125), - soit sous la forme du "gouffre obscur», d'où Baudelaire lance son «De profundis clamavi» (1975: 32).

Etrangers devant eux-mêmes, et devant la société qui les entoure, les deux écrivains avouent le sentiment d'un vide existentiel que ni les modèles du passé ni les propositions modernes ne parviennent à combler. La prise de conscience de leur marginalité en est d'autant plus douloureuse. Dans la lettre à sa mère datée du 3 août 1838, Baudelaire constate: «Rien de certain parmi les anciens, rien de beau parmi les modernes. Le passé et le présent sont deux statues incomplètes: l'une a été retirée toute mutilée du débris des âges; l'autre n'a pas encore reçu sa perfection de l'avenir» (1973, I: 61); dans le chapitre «L'avenir. Difficulté

7 Nous empruntons le sous-titre de la partie 2 du Livre quarante-quatrième des Mémoires: "Le passé. Le vieil ordre européen expire." 
de le comprendre», du Livre quarante-quatrième des Mémoires, Chateaubriand s'exprime en ces termes sur son époque: "Le monde actuel, le monde sans autorité consacrée, semble placé entre deux impossibilités: l'impossibilité du passé, l'impossibilité de l'avenir» (922); plus loin, dans "Récapitulation de ma vie», il reviendra sur l'entre-deux qui caractérise sa situation, tempérée quand même par l'espérance, la foi qui ne l'a pas abandonné devant l'incertitude de l'avenir, à l'inverse de Baudelaire qui, devenu aphasique à la fin de sa vie, en est devenu par trop «Voyant»: "Je me suis rencontré entre deux siècles, comme au confluent de deux fleuves; j'ai plongé dans leurs eaux troublées, m'éloignant à regret du vieux rivage où je suis né, nageant avec espérance vers une rive inconnue» (Chateaubriand, 1951: 936). C'est encore dans la lettre à sa mère, déjà citée, que Baudelaire avoue sa détresse envers la littérature contemporaine: «Je n'ai lu qu'ouvrages modernes [...] je suis complètement dégoûté de la littérature [qu'il considère le] terrain pourri de la fatuité moderne», et, sur le ton radical que son jeune âge autorisait, sa décision de ne plus rien lire.

La conscience de l'histoire non plus comme un mouvement unitaire vers l'avenir, mais plutôt comme une crise - dont la manifestation la plus sensible, fut, pour Baudelaire, la rupture entre l'idée de progrès et celle de ses corrélats, hérités du xvıIe siècle: l'idée de perfectibilité de l'homme et du bonheur, que la réalité dément -, conduira le poète vers l'évocation d'un paradis perdu; le spleen traverse son ouvre. A la nostalgie d'une histoire unifiante répond la fracture de l'histoire (Vattimo, 1990). Le fonds d'optimisme aux tonalités fouriéristes du Salon de 1846, dédié aux Bourgeois, est démenti dans Mon coeur mis à nu, dont le projet d'écriture ne doit pas remonter au-delà de 1859 (Baudelaire, 1975: 1491), année de Le peintre de la vie moderne.

Le vide de la modernité est encore ressenti par d'autres contemporains de Baudelaire, dont Flaubert, qui partage avec le poète sa haine tout aussi aristocratique du «bourgeois», dans un monde où il n’y a plus de place pour le poète, pour l'art ni pour le rêve. Parmi cette longue liste de désenchantés se côtoient des personnages fictifs tels Joseph Delorme, Samuel Cramer ou Emma Bovary, et des personnages réels, dont l'inconnu Constantin Guys. C'est aussi de cette période que date l'écart, de plus en plus sensible, entre l'artiste et la société, sa marginalité, son excentricité, pour utiliser l'expression de Daniel Sangsue (1987). Le sentiment de rupture informe la pensée de la modernité; pour Daniel 
Riou, la pensée de la modernité se fonde sur une "attitude philosophique, artistique, intellectuelle ou morale» (1998: 10). Cette attitude nous permet d'identifier des visions du monde convergentes entre nos deux auteurs, au-delà des contingences de nature historique et d'époque qui ont marqué leur vie et qui ont produit leur œuvre; contingences qui ont également dicté des formes de discours spécifiques.

Chateaubriand et Baudelaire, deux auteurs qui questionnent la modernité: modernes, dans la mesure où ils questionnent leur contemporanéité; antimodernes, dans la mesure où ils refusent certains engagements, au nom d'une clairvoyance prémonitoire, et de la quête de valeurs que la modernité ne leur apporte pas, les renvoyant à des ailleurs qui leur sont impossibles à rejoindre. Pour Chateaubriand, une quête qui a commencé par se manifester au niveau du déplacement, du voyage. Voyage dont l'écrivain a fait l'expérience multiple, tout autant que ses personnages, partageant avec lui l'émergence de l'autofiction. Cette quête conduit Chateaubriand vers des lieux et des moments sublimes, du crépuscule du matin au crépuscule du soir (pour reprendre des titres baudelairiens), dans des régions où l'exotisme était encore possible. Son errance acquiert alors valeur en elle-même, elle n'est plus comprise comme le passage du passé à un avenir rassurant; elle se solde souvent par la mort du héros. Chez Baudelaire, à l'opposé, le voyage refuse le mouvement, le déplacement. Son espace propre est celui de l'imaginaire du poète, l'imagination étant pour lui la reine des facultés; voyage intérieur, il préfigure les espaces artificiels (et l'artifice est l'un des motsclefs de la poétique baudelairienne) créés par Des Esseintes en 1884, pour qui, de même, «l'artifice paraissait [...] la marque distinctive du génie de l'homme» (Huysmans, 1977: 103).

Les deux auteurs font l'expérience d'une quête inassouvie: errance pour l'un, création d'espaces imaginaires pour l'autre. La quête devient leur lieu commun, le carrefour d'une rencontre spirituelle et poétique, celle de leur inquiétude, celle de leur contestation, de leur insatisfaction permanente, celle de leur exigence aussi.

Chateaubriand publie son premier livre en 1790, dans un genre alors à la mode: l'idylle, dans l'Almanach des Muses: l'Amour de la Campagne (un amour que Baudelaire ne partagera pas avec Chateaubriand, lui qui avait en horreur 
"l'état de nature»). Revue, titre et genre on ne peut plus romantiques au lendemain d'une révolution aussi profonde et bouleversante que celle qui venait de commencer. Son dernier titre sera publié lorsque Baudelaire aura 23 ans, La vie de Rancé (1844). Baudelaire a publié son premier salon en 1845; auparavant, des poèmes de jeunesse, anonymes ou en collaboration, avaient paru, de lui, dans l'Artiste. L'ombre de Chateaubriand plane sur l'œuvre de Baudelaire, le rôle de ce mâ̂tre à penser est aussi réel pour lui que celui de Joseph de Maistre et d'Edgar Allan Poe, qui lui «ont appris à raisonner» tel qu'il l'avoue dans ses écrits fragmentaires Hygiène, Fusées (1975: 669). Quelques poèmes de Baudelaire illustrent cette filiation.

La référence à René dans le poème de jeunesse, très probablement écrit en 1844 ou $1845^{\circ}$, que Baudelaire dédie à Sainte-Beuve, est en cela élucidative:

"- Le breuvage infiltré, lentement, goutte à goutte,

En moi qui dès quinze ans vers le gouffre entraîné,

Déchiffrais couramment les soupirs de René.» (207)

L'écho de Chateaubriand résonne également dans les derniers quatrains du poème Les Phares, probablement écrit à la fin de l'année 1855, couronnant le florilège des artistes précédemment cités dans ce poème.

A la mort de Delacroix (1863), Baudelaire évoque les sentiments qu'il éprouva en souvenir de Chateaubriand, Balzac et Vigny, dans l'éloge funèbre du peintre, "L'œuvre et la vie de Delacroix»: "nous avons tous senti quelque chose d'analogue à cette dépression d'âme, à cette sensation de solitude croissante que nous avaient fait déjà connaître la mort de Chateaubriand et celle de Balzac, sensation renouvelée tout récemment par la disparition d'Alfred de Vigny» (1976: 769). Les références croisées à Delacroix, Chateaubriand, Balzac, Vigny obligent à un détour par le palimpseste baudelairien. Le sens même de cette expression est clairement exposé par le poète, appliqué au processus même de la "création" artistique?:

8 Selon Claude Pichois (Baudelaire, 1975: 1237).

9 Pour Baudelaire, tel que la lecture de ses salons l'illustre, les expressions d' "artiste" et de "poète" se recoupent, toutes deux renvoyant à la création. 
"Un bon tableau, fidèle et égal au rêve qui l'a enfanté, doit être produit comme un monde. De même que la création, telle que nous la voyons, est le résultat de plusieurs créations dont les précédentes sont toujours complémentées par la suivante; ainsi un tableau conduit harmoniquement consiste en une série de tableaux superposés, chaque nouvelle couche donnant au rêve plus de réalité et le faisant monter d'un degré vers la perfection» (626).

Baudelaire fait clairement comprendre dans ce passage, extrait de son Salon de 1859, sa propre méthode de composition, son "art poétique». L'éloge funèbre de Delacroix et le texte de ce Salon s'inscrivent dans une ligne de continuité avec les Salons qui les précèdent, respectivement le Salon de 1845 et celui de 1846, démontrant ainsi la cohérence de la pensée esthétique baudelairienne. Par ailleurs, ces textes démontrent également la cohérence de la position du poète face à la modernité. L'ironie qu'il manifeste face à cette problématique se nourrit de sentiments ambivalents auxquels sa poétique nous a habitués, où le sentiment du spleen côtoie ses propos sarcastiques. Dans l'article intitulé «De l'essence du rire», le poète attire l'attention du lecteur sur la spécificité paradoxale de «ce genre singulier» - la caricature -, qu'il caractérise par «'introduction de cet élément insaisissable du beau jusque dans les œuvres destinées à représenter à l'homme sa propre laideur morale et physique». C'est ainsi qu'il rappelle les "grands symptômes moraux et littéraires" de la "comédie de Robert Macaire», que ses contemporains semblent ignorer (525-526). Déjà dans les Salons de 1845 et de 1846, Baudelaire déplorait l'aveuglement des «bourgeois» (auxquels il dédie ce dernier Salon) devant la modernité, devant les symptômes de «'hérö̈sme de la vie moderne» ${ }^{10}$, miroirs de la décadence, auxquels avaient également été sensibles Chateaubriand, Delacroix et d'autres contemporains. La série des Robert Macaire ${ }^{11}$ démontre bien, pour Baudelaire, dans quelle mesure le sarcasme est le symptôme de la conscience aiguë de cette modernité. Le port de l'habit noir ${ }^{12}$ révèle, aux yeux du poète, l'état moral et politique de son époque:

\footnotetext{
${ }^{10}$ Le chapitre XVIII du "Salon de 1846" s'intitule "De l'hérö̈sme de la vie moderne".

11 Robert Macaire, personnage imaginaire que le trait d'Honoré Daumier immortalisa dans la série Cent et un Robert Macaire, d'après la suggestion du directeur du journal humouristique, Charivari, Charles Philipon, em 1836.

12 Aussi célébré par Balzac, Sainte-Beuve dans Volupté ou dans la figure de Joseph Delorme, ou le Hugo des Chants du crépuscule.
} 
"Remarquez bien que l'habit noir et la redingote ont non seulement leur beauté politique, qui est l'expression de l'égalité universelle, mais encore leur beauté poétique, qui est l'expression de l'âme publique; - une immense défilade de croque-morts, croque-morts politiques, croque-morts amoureux, croque-morts bourgeois. Nous célébrons tous quelque enterrement» (494).

La modernité est ainsi caractérisée, d'après Baudelaire, par des allusions récurrentes, dans ses écrits, au sentiment de "solitude croissante», à un "deuil» dont la dimension est nationale, à l' "affaiblissement de la vitalité générale», à l'«obscurcissement de l'intellect», à l' "éclipse solaire» (et le thème solaire est un oxymoron de la pensée baudelairienne, à l'instar de la pensée romantique ${ }^{13}$ ). Dans ce contexte, que les réflexions écrites à l'occasion de la mort de Delacroix développent ${ }^{14}$, la séparation est d'autant plus accentuée entre les poètes et les artistes - élite unie dans la seule famille qui puisse l' intéresser, celle des «relations intellectuelles» -, et les "autres citoyens», cette foule (annonçant la massification future, conséquence néfaste de la "démocratie» entrevue par Chateaubriand) dont aussi bien Honoré Daumier que Baudelaire ont si bien su capter les traits caractéristiques. Toujours dans le même texte, malgré le ton de circonstance de sa clôture, nous pouvons reconnaître le rôle majeur que Baudelaire attribue à l'art, celui d'avertir ses contemporains lors de la disparition d'un artiste - "phare» nécessaire au cheminement de l'humanité:

"Quant aux autres citoyens, pour la plupart, ils n'apprennent que peu à peu à connaître tout ce qu'a perdu la patrie en perdant le grand homme, et quel vide il fait en la quittant. Encore faut-il les avertir» (769).

Les allusions explicites de Baudelaire à Chateaubriand sont fréquentes, davantage dans ses textes critiques que directement dans ses poèmes. Elles assument souvent une tonalité évocatoire, sinon même invocatoire, et témoignent de la présence incontournable de l'auteur d'Atala et de René ou des Natchez, des Mémoires d'outre-tombe et du Génie du Christianisme dans la pensée esthétique moderne. A commencer par cette brève référence au style de Chateaubriand,

\footnotetext{
13 V. Ambrière, Madeleine, Au soleil du romantisme, Paris, PUF, 1998.

14 En particulier dans le chapitre VIII.
} 
dans ce fragment de Fusées, auquel Baudelaire attribue dès lors la dimension supra-temporelle et universelle exemplaire qu'il cherche lui-même: «Style. La note éternelle, le style éternel et cosmopolite. Chateaubriand, Alph. Rabbe, Edgar Poe» (1975: 661). Le sentiment de la correspondance, ou de l'analogie universelle ${ }^{15}$ des arts, rapproche Chateaubriand et Delacroix, que l'admiration commune de Baudelaire réunit. Les références au "ton mélancolique du poète des Martyrs» sont évoquées dans ce passage du Salon de 1859 pour caractériser le style de Delacroix, à l'aide de l'inscription de quelques passages d'Atala dans son propre texte:

"Le ton mélancolique du poète des Martyrs s'adapte à ce tableau [la Montée au Calvaire], et la tristesse languissante du prisonnier chrétien s'y réfléchit heureusement. Il y a là l'ampleur de touche et de sentiments qui caractérisait la plume qui a écrit Les Natchez; et je reconnais, dans la sauvage idylle d'Eugène Delacroix, une histoire parfaitement belle parce qu'il y a mis la fleur du désert, la grâce de la cabane et une simplicité à conter la douleur que je ne me flatte pas d'avoir conservées ${ }^{16}$ (1976: 635-636).

Par ailleurs, et pour ne faire référence qu'à quelques échos de cette présence titulaire, nous constatons que le sentiment de nostalgie qu'éveille en Baudelaire le souvenir de Chateaubriand localise celui-ci en un temps hors du temps et de l'espace, voire un temps mythique. Souvent aussi, l'adjectivation utilisée par Baudelaire l'entoure d'une image grandiose. C'est le cas de ce passage du poème en prose La chambre double: "La chambre paradisiaque, l'idole, la souveraine des rêves, la Sylphide, comme disait le grand René, toute cette magie a disparu au coup brutal frappé par le Spectre» (1975: 281); c'est encore le cas de l'essai Un mangeur d'opium, lorsque, dans le chapitre intitulé «Le génie enfant", Baudelaire, qui commente l'œuvre de Thomas de Quincey, se référant «aux différentes métaphores dont se servent les poètes pour peindre l'homme revenu des batailles de la vie», recourt encore à Chateaubriand: "C'est ce que, d'une manière générale, j’appellerais volontiers le ton du revenant; accent, non pas surnaturel,

15 Les deux expressions s'équivalent dans la poétique baudelairienne.

16 Tel que Claude Pichois le confirme, les «expressions en italique sont empruntées à l'Epilogue d'Atala." (1976: 1397). 
mais presque étranger à l'humanité, moitié terrestre et moitié extra-terrestre, que nous trouvons quelques fois dans les Mémoires d'outre-tombe, quand, la colère ou l'orgueil blessé se taisant, le mépris du grand René des choses de la terre devient tout à fait désintéressé» (496). Des réflexions d'ordre moral traversent également son ouvre, tel qu'il arrive dans la transcription d'un passage des Mémoires d'outre-tombe dans un fragment de ses Journaux intimes, dans la partie Hygiène, intitulé "Hygiène, morale, conduite», contenant des réflexions sur les effets du passage du temps dans la vie de l'homme (671). L'expérience ressentie par Chateaubriand, tout aussi déçu par les peuples primitifs que par la société contemporaine, situé entre deux mondes incompatibles, entre l'«impossibilité du passé» et l' «impossibilité de l'avenir» annonce, en elle-même une situation d'impasse, elle semble devancer ce que des historiens, après Hegel, ont désigné comme la fin de l'bistoire ${ }^{17}$. Dans le chapitre "L'avenir», du livre quarante-quatrième des Mémoires, la conscience de la fin d'un temps messianique s'accompagne de l'ironie avec laquelle Chateaubriand fait référence à la nouvelle foi des temps modernes, la science: "Il ne resterait qu'à demander à la science le moyen de changer la planète». La croyance au progrès, basée sur la même foi, est également contestée par Baudelaire, pour qui la science, le progrès, justement parce qu'ils cherchent à dévoiler l'inconnu, nuisent à l'imagination, au mystère dont s'entoure l'art. Le rêve d'un artiste qui ne peint que selon sa sensibilité ressort des textes où Baudelaire s'interroge sur le réalisme, sur la photographie; dans le Salon de 1859, faisant l'apologie de l'imagination, unique source de l'art véritable, il s'exprime sans ambiguïté: "L'artiste, le vrai artiste, le vrai poète, ne doit peindre que selon ce qu'il voit et qu'il sent» (1976: 620). Rejoignant Rimbaud, pour qui la "vraie vie est ailleurs», Baudelaire établit la rupture avec l'art (les arts) représentatif(s): "Je trouve inutile et fastidieux de représenter ce qui est, parce que rien de ce qui est ne me satisfait. La nature est laide, et je préfère les monstres de ma fantaisie à la trivialité positive» (620). Refusant de comprendre et de peindre le réel décevant, Baudelaire ouvre la voie à Proust, pour lequel c'est également l'expérience esthétique qui permet d'organiser le monde. En 1863, à peine quatre ans avant la disparition du poète, Jules

17 Pour l'histoire du concept de "fin de l'histoire», v. Bourgeois, Bernard, "La fin de l'histoire", in La raison moderne et le droit politique, Paris, Vrin, 2001. 
Verne travaillait à son roman (publié posthumément), Paris au $x x^{e}$ siècle, dans lequel il présente un Paris complètement rendu à la science et à la technique, où les hommes sont prêts à devenir des robots, et où l'art et la littérature n'ont plus de place, si ce n'est que pour célébrer la science. C'est ainsi que le héros de ce roman, s'étant rendu un jour à la librairie la plus moderne de Paris, la Librairie des Cinq Parties du Monde, afin d'acheter quelques livres de poètes consacrés, tels Hugo, Lamartine, Musset, a pu constater que leur œuvre et leur nom étaient complètement inconnus des fonctionnaires qui l'avaient accueilli; par contre, il s'est vu proposer l'achat de quelques «œuvres littéraires contemporaines», parmi les «quelques productions qui [avaient] fait un certain bruit ces dernières années» et s'étaient bien vendues "pour des livres de poésie», dont, "entre autres, les Harmonies Electriques de Martillac, ouvrage couronné par l'Académie des Sciences, les Méditations sur l'oxygène de M. de Pulfasse, le Parallélogramme poétique, les Odes décarbonatées...» (Verne, 1994: 60). Il suffirait de rappeler que Maxime du Camp, contemporain de Baudelaire, avait publié ses Chants modernes, en 1855, en honneur de la vapeur, du chloroforme, de l'électricité et des locomotives... précédés d'une préface qu'il souhaitait voir reconnue comme le parallèle moderne de la Préface de Cromwell au moment où Hugo l'avait publiée, en 1827 - véritable manifeste romantique en France, inaugurant la rupture avec les dogmes classiques.

La conception de la notion de progrès de Chateaubriand et de Baudelaire s'oppose à la définition qu'en donne le Grand dictionnaire universel du xIxe siècle, de Pierre Larousse: "la marche du genre humain vers la perfection, vers son bonheur: l'humanité est perfectible et elle va incessamment du moins bien au mieux, de l'ignorance à la science, de la barbarie à la civilisation».

La figure du progrès ainsi comprise nous aide à mieux comprendre comment prennent corps les concepts-clés de perfectibilité/réversibilité, progrès/bonheur, ignorance/science, barbarie/civilisation à la lumière des figures de l'antimodernité données par Compagnon.

Attardons-nous sur l'impossibilité de croire au progrès comme source de bonheur et garant de pérennité chez Baudelaire, sur la problématique de sa croyance au péché originel. Tel que Compagnon le rappelle, "contre la métaphysique moderne du progrès, Baudelaire réaffirme la théologie du péché 
originel, fondement du mal universel» (88). Cette figure s'annonce en opposition aux thèses de Rousseau, en particulier à ce que Fabienne Bercegol nomme "l'idéologie dominante» en faveur de la thèse de la "perfectibilité»"18 humaine; en opposition à l'idée de nature comme fondement de la morale au xvine siècle. Pour Baudelaire, toute l' "hérésie» est là, d'avoir remplacé «lidée du péché originel» comme fondement du comportement humain, par celle d'une nature d'où le péché serait absent. La lettre qu'il adresse le 21 janvier 1856 à Toussenel, ardent propagateur de la doctrine de Fourier, élucide le lecteur de la correspondance du poète sur ce point.

Fabienne Bercegol, dans l'article intitulé «Chateaubriand ou la conversion au progrès», rend compte de la polémique lancée par l'écrivain «en 1797, contre les tenants de l'idée de progrès, contre cette lignée de philosophes qui, de Fontenelle à Condorcet, se sont efforcés de renouveler la compréhension de l'histoire humaine, en substituant au modèle théologique ou cyclique, un schéma linéaire, qui la présente comme un mouvement global et continu, orienté vers un progrès dont l'homme est le moteur» (26).

Ainsi, Chateaubriand écrit dans l'Essai sur les révolutions:

"On pourrait soupçonner qu'il existe des époques inconnues, mais régulières, auxquelles la face du monde se renouvelle. Nous avons le malheur d'être nés au moment d'une de ces grandes révolutions: quel qu'en soit le résultat, heureux ou malheureux pour les hommes à naître, la génération présente est perdue» (1978: 72).19

On comprendra mieux, à partir de la pensée de l'auteur des Essais sur les révolutions (1797) le malaise ressenti par Baudelaire face à son époque, de même que l'importance - et le pouvoir - dont se revêt l'histoire pour éclairer l'avenir. Nous retrouvons chez Baudelaire des échos de la fidélité de Chateaubriand «à la vision cyclique d'une histoire faite de recommencements" s'opposant à la "capacité naturelle de l'homme à progresser et [à] la possibilité d'un progrès général

18 Tel que l'explique Bercegol, la notion de "perfectibilité", introduite par Rousseau dans son Discours sur l'origine et les fondements de l'inégalité parmi les hommes (1755), est interprétée négativement par l'auteur: "en faisant de cette aptitude au perfectionnement progressif une propriété virtuelle de l'homme qui se révèle finalement néfaste, dans la mesure où elle hate sa déchéance morale" (Bercegol, 2000: 26).

19 Ce passage est plus longuement transcrit par Bercegol dans l'article cité, p. 26 
par la connaissance» (Bercegol, 2000: 26). Dans les Essais, Chateaubriand se démarque progressivement des théories de la "perfectibilité continue»; il accentuera sa pensée sur la dégénérescence, le sentiment du manque et la mélancolie que nous retrouverons chez Baudelaire, et sur lesquels il semble revenir dans la dernière partie des Mémoires. Chateaubriand se rapproche à ce moment de Rousseau, à l'opposé des philosophes des Lumières; pourtant, il va plus loin que Rousseau, car il introduit le christianisme comme moteur de la modernité et source de perfectionnement, dans Le Génie du Christianisme, ouvrage auquel il travailla entre 1795 et 1799 pendant son exil en Angleterre et qu'il publia en 1802, alors qu'il se trouvait déjà en France. Baudelaire se radicalise après 1848, il lira les Mémoires et les Essais. A l'instar du Chateaubriand des Essais, il ne croira plus à aucune forme de gouvernement fondé sur la démocratie, tel qu'il le dit avec conviction dans ce fragment de Mon coeur mis à nu: «Il n'y a de gouvernement raisonnable et assuré que l'aristocratique. Monarchie ou république basées sur la démocratie sont également absurdes et faibles» (684). Nous lisons dans les Essais: "Tout gouvernement est un mal», "tout se réduit à l’indépendance individuelle» (269; 270). La défense de l'indépendance individuelle mène chez Chateaubriand au désir de se "faire sauvage»; chez Baudelaire à s'identifier en tant que poète, artiste, créateur, à Dieu. Dans l'essai Exposition universelle (1855), l'un de ses textes les plus importants pour la compréhension de l'idée de modernité, on peut lire: "L'artiste ne relève que de lui-même. Il ne promet aux siècles à venir que ses propres œuvres. Il ne cautionne que lui-même. Il meurt sans enfants. Il a été son roi, son prêtre et son Dieu» (1976: 581).

Si la notion de péché originel, au sens où Baudelaire l'emploie, empêche chez lui toute possibilité de progrès ou de perfectibilité, chez Chateaubriand, par contre, surtout dans le Génie du christianisme, cette notion permet de comprendre la condition humaine. Selon Fabienne Bercegol, «il faudra attendre le Génie, et donc le retour au religieux, pour qu'il sorte véritablement de l'impasse où l'avait conduit son nihilisme politique et redonne toute leur place au progrès et à la liberté dans l'histoire des hommes» (33).

Pour Chateaubriand, donc, la réconciliation est possible entre politique et religion, le christianisme nourrissant la modernité dans sa soif de liberté et d'égalité; chez Baudelaire, sa poétique célèbre au contraire l'agressivité, à l'image des arabesques que trace la "sinuosité du verbe» de ses Petits poèmes en prose. 
L'expression «théologie du progrès», proposée par Bénichou dans Le Temps des prophètes, conviendrait ainsi à la "conversion" de Chateaubriand; déjà pour Baudelaire, faudra-t-il parler d'une «théologie destructrice, orphique, désespérée», qui s'abat - l' "action d'éclat" accomplie par le poète dans le petit poème en prose Mauvais vitrier en est un exemple bien réussi - sous le soleil noir du "Sacrifice de l'innocent au profit du coupable». "Effet pervers", la formule de la "réversibilité» proposée par De Maistre, illustre bien «cette inversion du mal en bien» (Compagnon, 2005: 64). Et Compagnon de conclure: «Pour l'antimoderne, on est toujours coupable et le mal est partout» (104); victime et bourreau, le poème en prose l' "Héautontimorouménos» illustre cette conséquence du péché originel, susceptible de conduire au «déplacement de la vitalité», à la «dissociation de la volonté» de l'homme moderne, solitude et absence de continuité que partagent Baudelaire et Chateaubriand. C'est ainsi que Baudelaire donnera sa "théorie de la vraie civilisation»: pour le poète, «elle n'est pas dans le gaz, ni dans la vapeur, ni dans les tables tournantes, elle est dans la diminution des traces du péché originel» (1975: 697). Toujours dans Mon cour mis à nu, il soutiendra que "la croyance au progrès est une doctrine de paresseux", qu' "il ne peut y avoir de progrès (vrai, c'est-à-dire moral) que dans l'individu et par l'individu lui-même» (681). Ainsi s'explique sans doute le qualificatif de «mauvais» appliqué au vitrier, dans le poème en prose Le mawvais vitrier: ayant pris en horreur le sens de l'utile qui s'est emparé de la civilisation bourgeoise, Baudelaire punit en ce "pauvre homme» son métier, qui l'absorbe et l'empêche de voir au-delà des vitres ${ }^{20}$ mêmes - malgré leur transparence - qu'il transporte toute la journée afin d'assurer sa subsistance. Fils de la modernité urbaine, condamné à se fondre dans la grande masse laborieuse, il est de ce fait incapable d'accéder à la beauté, à l'idéal, à l'art. De même, n'y a-t-il plus de place faite au poète dans la ville moderne; en ce sens, le vitrier et le poète sont tous deux forcés de se tenir en marge de la société, ils partagent le même sort. La violence décrite dans ce poème et le sarcasme sur lequel il se termine, ne s'abattent pas en exclusivité sur le vitrier: ils atteignent également le poète lorsqu'il sombre sous la médiocrité, lorsqu'il se révèle incapable d'atteindre l'Idéal, se reconnaissant, au fond, tout proche de lui par son travail, même s'il a honte de le reconnaître, lui qui n'aime

20 Le thème des fenêtres étant un des thèmes majeurs de la poétique baudelairienne. 
pas à écrire des préfaces, lui qui est si réticent à assumer la poésie comme un métier, son métier ${ }^{21}$. La modernité met fin à l'harmonie des correspondances, à l'allégorie, la chambre du poète ne se teinte "de rose et de bleu» que par de brefs instants; «un coup terrible, lourd" mettra fin à la "magie» qui l'enveloppait et lui fera prendre conscience de la double réalité. Il n'y aura plus de place au "culte des images" dans la société moderne; la foule court à sa perte, l'humanité semble condamnée et des échos de De Maistre se font entendre sous le pessimisme baudelairien.

Cependant ce n'est pas uniquement en termes sociaux ou historiques que le lecteur est invité à lire Baudelaire aujourd'hui.

La morale de la violence préside à bien d'autres poèmes baudelairiens, Assommons les pauvres, Le Voyage, Le Cygne, Le Gâteau, Le Soleil ou Duellum, parmi d'autres. Elle devient alors l'allégorie du travail poétique, acte générateur du poème lui-même. C'est ainsi que le travail poétique est souvent chez Baudelaire défini par un recours à un vocabulaire du domaine militaire: les expressions de "lutte", d'"escrime", témoignent de la résistance soit sociale, soit poétique, qu'il doit affronter dans la modernité.

Rappelons-en son dernier vou - son programme poétique de la modernité à la fin de Les Fleurs du Mal:

«Plonger au fond du gouffre, Enfer ou Ciel, qu'importe?

Au fond de l'Inconnu pour trouver du nouveau!»

Mais, comme Chateaubriand, Baudelaire sort-il vainqueur de sa quête d'un monde nouveau, d'une poétique nouvelle?

A force de vouloir "éterniser l'ardeur de [leur] haine», les deux guerriers du poème Duellum se condamnent mutuellement - nouvelle figure du poète, double, bourreau de soi-même -; de leur descente au gouffre naîtra le poème, la fleur du mal:

"Dans le ravin hanté des chats-pards et des onces

Nos héros, s'étreignant méchamment, ont roulé,

Et leur peau fleurira l'aridité des ronces.»

21 Franc Schuerewegen analyse la problématique du travail d'écrivain, chez Baudelaire, dans son article "De l'inexistence de l'art: Genèse des Fleurs du mal» publié en 1998. 
Rappelons-nous le Chateaubriand de la fin des Mémoires, lucide devant la marche inexorable de l'humanité vers l'abîme...

Baudelaire nous réapprend à lire Chateaubriand, qu'il resitue face à modernité antimoderne. En revenant à la proposition initiale de notre étude, nous dirons avec Leyla Perrone-Moisés (12), traductrice de Borges, que:

Cada escritor cria seus precursores. Seu labor modifica nossa concepção do passado, como há-de modificar o futuro

(Leyla Perrone-Moisés, 1984) 


\section{BIBLIOGRAPHIE}

BAUDELAIRE (1973), Correspondance, Gallimard, 2 vols. (1975-1976), Euvres complètes, Gallimard, I-II.

BARBERIS, Pierre (1976), Chateaubriand: une réaction au monde moderne, Larousse.

BARTHES (1994), "Critique et vérité», Euvres complètes, Seuil, II.

BENICHOU, Paul (1977), Le temps des prophètes, Gallimard.

BERCEGOL, Fabienne (2000), "Chateaubriand ou la conversion au progrès", Romantisme, 108: 23-51.

CHATEAUBRIAND (1951), Mémoires d'outre-tombe, Gallimard, 2 vols. (1969), Euvres romanesques et voyages, Gallimard. René: 117-146. (1978), Essais sur les révolutions. Génie du Christianisme, Gallimard.

COMPAGNON (2005), Les antimodernes: de Joseph de Maistre à Roland Barthes, Gallimard. HUYSMANS (1977), A rebours, Gallimard.

PERRONE-MOISES, Leyla (1984), «História literária e julgamento de valor» Colóquio-Letras, 77: 5-18.

REVERSY, Eléonore (2009), "Madame Bovary, 1858: Fanny, d'Ernest Feydau, savoirs de l'écriture», in REY, Pierre-Louis; SEGINGER, Gisèle, Madame Bovary et les savoirs, Presses de la Sorbonne Nouvelle: 273-282.

RIOU, Daniel (1998), "De la Modernité», Euvres et critiques, XXIII, 1: 9-27.

SANGSUE, Daniel (1987), Le récit excentrique, José Corti.

SARTRE, (1948), Qu'est-ce que la littérature?, Gallimard.

SCHUEREWEGEN, Franc (1998), "De l'inexistence de l'art: genèse des Fleurs du Mal», Poétique, 114: 131-140.

STENDHAL, Racine et Shakespeare, Libraire Hatier: http://gallica.bnf.fr/ark:/12148/ bpt6k6148004p/f25.image.r=.langFR, consulté le 30 décembre 2011.

VATTIMO, Gianni (1990), La société transparente, Desclée de Brouwer.

VERNE, Jules (1994) Paris au XXe siècle, Hachette. 